\title{
Acute exacerbations of chronic obstructive pulmonary disease provide a unique opportunity to take care of patients
}

\author{
Bianca Beghé, ${ }^{1}$ Martina Garofalo, ${ }^{2}$ Leonardo M. Fabbri ${ }^{3}$ \\ ${ }^{1}$ Clinica di Malattie dell'Apparato Respiratorio; ${ }^{2}$ Dipartimento di Oncologia, Ematologia e Patologie dell'Apparato Respiratorio, \\ Azienda Ospedaliero-Universitaria di Modena; ${ }^{3}$ Università degli Studi di Modena e Reggio Emilia, Modena, Italy
}

\begin{abstract}
Exacerbation of chronic obstructive pulmonary disease (ECOPD) identifies the acute phase of COPD. The COPD patient is often frail and elderly with concomitant chronic diseases. This requires the physician not only looks at specific symptoms or organs, but to consider the patient in all his or her complexity.
\end{abstract}

\section{Comment to Management of exacerbations of chronic obstructive pulmonary disease with a focus on comorbidities by Jadwiga A. Wedzicha}

The term exacerbation of chronic obstructive pulmonary disease (ECOPD) identifies the acute phase of COPD, i.e. the presentation of symptoms due to the worsening of the characteristic chronic airway and/or pulmonary inflammation associated with the disease. ${ }^{1}$ Events that fall within the term ECOPD are, for example, viral or bacterial respiratory infections during which the inflammatory process triggered by the infection overlaps with the chronic inflammation and results in a worsening of the typical symptoms of COPD, i.e. dyspnea, cough, and sputum. Mechanisms are stimulation of nerve reflexes, bronchial and vascular congestion, purulent secretions, bronchocon-

Correspondence: Leonardo M. Fabbri, Università degli Studi Modena e Reggio Emilia, Via del Pozzo 71, 41100 Modena, Italy.

Tel. +39.059.4224198/4222198 - Fax: +39.059.4224231.

E-mail: leonardo.fabbri@unimore.it

Key words: chronic obstructive pulmonary disease, exacerbation, complex patient, respiratory and non-respiratory mechanisms, comprehensive treatment.

This work is licensed under a Creative Commons Attribution NonCommercial 3.0 License (CC BY-NC 3.0).

CCopyright B. Beghé et al., 2013

Licensee PAGEPress, Italy

Italian Journal of Medicine 2013; 7:82-83

doi:10.4081/itjm.2013.82 striction, and alteration of the ventilation/perfusion interaction in the lung. ${ }^{2}$.

In a short but concise and comprehensive review on ECOPD published in this issue of the Journal, ${ }^{3}$ Prof. Wedzicha, one of the most influent experts on COPD, describes the complexity not only of the ECOPD but also of the COPD patient. In fact, the COPD patient often suffers from concomitant chronic diseases, both respiratory (e.g. asthma, bronchiectasis, pulmonary fibrosis, etc.) and non-respiratory (hypertension, heart failure, ischemic heart disease, arrhythmia, gastroesophageal reflux, obesity, dyslipidemia, arteriosclerosis, osteoporosis, diabetes, depression, etc.).

Patients with COPD and concomitant chronic diseases are often elderly and frail, and are frequently at higher risk both of acute newly developed episodes (bronchitis, pneumonia, pulmonary embolism, pneumothorax, etc.) and of worsening of concomitant chronic disease (e.g. asthma and/or bronchiectasis exacerbation, hypertension, heart failure, myocardial ischemia, heart infarction, etc.). ${ }^{4}$ Prof. Wedzicha recommends that physicians and medical staff should always consider these complex issues when visiting and treating patients with ECOPD. A useful example that emphasizes the importance of this comprehensive approach comes from a recent study of the Federation of Associations of Hospital Doctors on Internal Medicine (FADOI) conducted in emergency units located in Lombardy, northern Italy, and reported in our Journal. ${ }^{5}$ This study showed that, in COPD patients coming to the emergency unit with worsening dyspnea, the cause was primarily due to heart failure, not to acute bronchitis. $^{5}$

The conclusions of Prof. Wedzicha's review are both novel and of importance: i) in most cases, ECOPD may not be just an exacerbation of COPD, but the result of the complex interaction between respiratory and non-respiratory mechanisms; ii) considering this complexity, the expression $E C O P D$ seems 
to be more and more restrictive, and a new terminology that also reflects complexity and severity should be developed; iii) the complexity of the involved mechanism leads us to believe that the treatment for ECOPD recommended by current guidelines ${ }^{1}$ (bronchodilators, steroids, antibiotics) only focuses on relieving respiratory symptoms. It seems that treatment is very limited and probably only marginally effective, and that a more comprehensive treatment should be provided to address potential concomitant nonrespiratory components (e.g. heart failure, thromboembolisms, etc.).

COPD exacerbations that lead to hospitalization have a poor prognosisis, ${ }^{6}$ with $28 \%$ risk of death in one year and $80 \%$ in nine years. ${ }^{7,8}$ The most frequent causes of death in patients with ECOPD are pulmonary embolism, heart failure, respiratory infections and respiratory failure. ${ }^{9,10}$

It is possible that, as happened in patients with myocardial infarction, a new comprehensive pharmacological and non-pharmacological treatment and its early application might improve the prognosis of ECOPD. This would only be possible if we not only look at specific symptoms or organs, but consider the patient in all his or her complexity.

\section{References}

1. Vestbo J, Hurd SS, Agusti AG, et al. Global strategy for the diagnosis, management and prevention of chronic obstructive pulmonary disease, GOLD executive sum- mary. Am J Respir Crit Care Med 2012. [Epub ahead of print].

2. Mackay AJ, Hurst JR. COPD exacerbations: causes, prevention, and treatment. Med Clin North Am 2012;96: 789-809.

3. Wedzicha JA. Management of exacerbations of chronic obstructive pulmonary disease with a focus on comorbidities. Ital J Med 2013;7:84-87.

4. Roca M, Verduri A, Corbetta L, et al. Mechanisms of acute exacerbation of respiratory symptoms in chronic obstructive pulmonary disease. Eur J Clin Invest 2013. [Epub ahead of print].

5. Minora T, Marino P. B- type natriuretic peptide and COPD in emergency department (ED). Ital J Med 2010; 4:247-53.

6. Suissa S, Dell'Aniello S, Ernst P. Long-term natural history of chronic obstructive pulmonary disease: severe exacerbations and mortality. Thorax 2012;67:957-63.

7. Slenter RH, Sprooten RT, Kotz D, et al. Predictors of 1-year mortality at hospital admission for acute exacerbations of chronic obstructive pulmonary disease. Respiration 2013;85:15-26.

8. Gudmundsson G, Ulrik CS, Gislason T, et al. Long-term survival in patients hospitalized for chronic obstructive pulmonary disease: a prospective observational study in the Nordic countries. Int J Chron Obstruct Pulmon Dis 2012;7:571-6.

9. McGarvey LP, Magder S, Burkhart D, et al. Cause-specific mortality adjudication in the UPLIFT ${ }^{\circledR}$ COPD trial: findings and recommendations. Respir Med 2012; 106:515-21.

10. Zvezdin B, Milutinov S, Kojicic M, et al. A postmortem analysis of major causes of early death in patients hospitalized with COPD exacerbation. Chest 2009; 136:376-80. 